\title{
Relativistic Accretion into a Reissner-Nordström Black Hole Revisited
}

\author{
J. A. de Freitas Pacheco \\ Laboratoire Cassiopée-UMR 6202, Observatoire de la Côte d'Azur, University of Nice-Sophia Antipolis, \\ BP 4222, 06304 Nice, Cedex 4, France \\ Correspondence should be addressed to J. A. de Freitas Pacheco, pacheco@oca.eu
}

Received 20 June 2011; Revised 26 September 2011; Accepted 28 September 2011

Academic Editor: Y. Peles

Copyright (๑) 2012 J. A. de Freitas Pacheco. This is an open access article distributed under the Creative Commons Attribution License, which permits unrestricted use, distribution, and reproduction in any medium, provided the original work is properly cited.

\begin{abstract}
The accretion of relativistic and nonrelativistic fluids into a Reissner-Nordström black hole is revisited. The position of the critical point, the flow velocity at this point, and the accretion rate are only slightly affected with respect to the Schwarzschild case when the fluid is nonrelativistic. On the contrary, relativistic fluids cross the critical point always subsonically. In this case, the sonic point is located near the event horizon, which is crossed by the fluid with a velocity less than the light speed. The accretion rate of relativistic fluids by a Reissner-Nordström black hole is reduced with respect to those estimated for uncharged black holes, being about $60 \%$ less for the extreme case (charge-to-mass ratio equal to one).
\end{abstract}

\section{Introduction}

The steady relativistic spherical flow of a perfect gas into a black hole has been intensely investigated in the past thirty years and a comprehensive review on the subject can be found, for instance, in [1]. For a Schwarzschild black hole, the basic relativistic equations of the inflow were discussed by Michel [2], who has derived the relations involving the sound and flow velocities at the critical point. These early investigations have shown that, for an adiabatic flow of a perfect gas with $\gamma<5 / 3$ into a Schwarzschild black hole, the only critical point of the flow lying outside the horizon is that corresponding to the Bondi solution. A similar situation occurs in the case of a weakly interacting gas supposed to model dark matter. Assuming that during the inflow the phase space density is conserved, the authors in [3] derived a solution for the critical point, which is located at a distance of about 30-150 times the horizon radius for conditions expected to be present in typical dark matter halos.

Under adiabatic conditions (the cooling time is longer than the free-fall timescale), the gas is compressed as it approaches the horizon, its temperature increases, and Xrays are emitted from the inner accreting envelope. This emission preheats the infalling gas, reducing considerably the accretion rate and the radiative efficiency [4]. Higher efficiencies can be obtained if dissipative turbulent motions and magnetic field line reconnection effects are included in the treatment of the inflow [5]. A consistent relativistic analysis of radiation effects on the flow was investigated in the pioneering work by Thorne and collaborators [6]. For an optically thick inflow, the distance of the critical point to the black hole horizon is reduced as well as the accretion efficiency [7]. In fact, when radiation effects are included, two branches appear in the diagram "luminosity versus accretion rate" $[8,9]$ but even in the most favourable cases, corresponding to the high luminosity branch, the radiation efficiency is at maximum $2 \times 10^{-4}$, three orders of magnitude smaller than the "canonical" value (of ten percent, derived from an accreting disk) usually adopted in cosmological simulations intended to explain the presence of supermassive black holes in the early universe by the growth of primordial seeds [10].

How the inflow properties are affected if the black hole has an electrical charge? Although the existence of charged black holes in the universe may be contested, some authors have hypothesized that such objects could play an important role in some astrophysical processes. For instance, 
the creation of positron-electron pairs in the "dyadosphere" of a Reissner-Nordström (RN) black hole was investigated in [11] (and references therein) as a possible mechanism to drive gamma-ray bursts. If the observed acceleration of the expansion of the universe is due to a phantom field, the interaction with a supermassive Schwarzschild black hole was analyzed in [12], while the interaction with an RN black hole was considered by $[13,14]$. As a consequence of this process, the mass of the black hole decreases but its charge remains constant and after a finite timescale, the black hole reaches the extreme state and may eventually produce a naked singularity [13].

As mentioned above, the formation of a charged black hole during the gravitational collapse and, in particular, the formation of a "dyadosphere" present several difficulties whose discussion is beyond the scope of the present paper (see, however, criticisms by [15]). Nevertheless, the accretion process by a charged black hole offers the possibility of studying different aspects of gravity and of accretion flows in extreme conditions. In the present paper, the accretion of fluids with different equations of state into an RN black hole is revisited. We will show that for a nonrelativistic baryonic fluid, the physical properties of the flow do not differ considerably from the Schwarzschild case, since the critical point is only slightly modified by the presence of a charge even in the extreme case. However, the situation is considerably different if the fluid is relativistic. In this case, the critical point is situated near the horizon and the flow velocity at this position is subsonic. Moreover, also the horizon crossing occurs at a subsonic velocity, contrary to what happens when the flow is nonrelativistic, case in which the horizon is crossed with a flow velocity equal to the light velocity. No solutions for the flow were found beyond the critical point if a naked singularity is present, confirming the conclusion by [13]. For both cases, relativistic and nonrelativistic flows, corrections to the accretion rate due to the presence of a charge are given. This paper is organized as follows: in Section 2, the equations of the flow are presented; in Section 3, the accretion of a nonrelativistic fluid is discussed, while the accretion of relativistic fluids is analyzed in Section 4. Finally, in Section 5, the main conclusions are given.

\section{Equations of the Accretion Flow}

The metric describing an RN black hole is given by

$$
d s^{2}=-B(r) d t^{2}+B^{-1}(r) d r^{2}+r^{2} d \omega^{2},
$$

where $d \omega^{2}=d \theta^{2}+\sin ^{2} \theta d \phi^{2}$ and the lapse function is defined by

$$
B(r)=1-\frac{r_{g}}{r}+\frac{Q^{2}}{r^{2}},
$$

where $r_{g}=2 M$ is the gravitational radius, $M$ and $Q$ are, respectively, the mass and the charge of the black hole. The zeros of the lapse function $B(r)$ define two horizons given by

$$
r_{ \pm}=\frac{r_{g}}{2}\left(1 \pm \sqrt{1-\beta^{2}}\right),
$$

and we have defined $\beta=Q / M$. The sign "+" corresponds to the outer or to the event horizon while the sign "-" corresponds to the inner horizon. When the charge satisfies the condition $Q=M$ (or, equivalently, $\beta=1$ ), both horizons coincide and this case corresponds to an extreme RN black hole.

We assume that a steady spherical inflow is set up inside the influence radius of the black hole, defined by the equality between the gravitational potential of the black hole and the mean kinetic energy of particles constituting the fluid far away from the horizon. Denoting by " $\nabla$ " the covariant derivative, the conservation equations are the mass flux conservation:

$$
\nabla_{k} J^{k}=0
$$

where $J^{k}=m n u^{k}$ is the mass-current density. The second equation is the energy-momentum flux conservation:

$$
\nabla_{k} T_{i}^{k}=0,
$$

where the energy-momentum tensor is that of an ideal fluid, that is, $T_{i}^{k}=(P+\varepsilon) u^{k} u_{i}-P \delta_{i}^{k}$, with $P$, $\varepsilon$, and $n$ being, respectively, the proper pressure, the proper energy density, and the proper particle number density. The only nonnull components of the 4 -vector velocity are $u=u^{1}=d r / d s$ and $u^{0}=d t / d s$. In the one hand, under spherical symmetry and steady state conditions, from the time-space component of (5) and the expression for the stress-energy tensor, one obtains

$$
(P+\varepsilon) u_{0} u^{1} \sqrt{-g}=C_{1},
$$

where $-g$ is the metric determinant of (1) and $C_{1}$ is an arbitrary integration constant. Using the normalization condition $u_{i} u^{i}=-1$, one obtains trivially that

$$
u_{0}=\left(1-\frac{r_{g}}{r}+\frac{Q^{2}}{r^{2}}+u^{2}\right)^{1 / 2} .
$$

On the other hand, the integration of the space component of (4) gives

$$
n u^{1} \sqrt{-g}=C_{2},
$$

where $C_{2}$ is another arbitrary integration constant. Substituting (7) into (6) and performing the ratio with (8) one obtains

$$
\frac{(P+\varepsilon)}{n}\left[1-\frac{r_{g}}{r}+\frac{Q^{2}}{r^{2}}+u^{2}\right]^{1 / 2}=\Delta,
$$

where $\Delta=C_{1} / C_{2}$ is a new constant. Notice that if $Q=$ 0 , one obtains the same result as in [2, equation (9)] but notice that here the particle number density $n$ appears in the denominator instead of the fluid energy density. Deriving (9) with respect to the radial coordinate $r$ and using the mass conservation equation (8), one obtains, after some algebra, the following equation:

$$
\frac{d \lg u}{d \lg r}\left[u^{2}-V^{2} F(r, u)\right]=\left[2 V^{2} F(r, u)-\left(\frac{r_{g}}{2 r}-\frac{Q^{2}}{r^{2}}\right)\right] .
$$


In the above equation, we have introduced, respectively,

$$
\begin{gathered}
F(r, u)=\left(1-\frac{r_{g}}{r}+\frac{Q^{2}}{r^{2}}+u^{2}\right), \\
V^{2}=\frac{d \lg (P+\varepsilon)}{d \lg n}-1 .
\end{gathered}
$$

The critical point of the flow occurs when both bracketed factors in (10) vanish simultaneously. It should be emphasized that the critical point, contrary to usual assertions found in the literature (see, e.g, [14]), does not necessarily coincide with the sonic point, in which a transition from subsonic to supersonic flow occurs. In fact, this is the situation occurring in outflows present in atmospheres of massive stars, driven by radiative forces $[16,17]$. For an accreting relativistic fluid, as we will see below, the crossing of the critical point always occurs while the flow is still subsonic.

The conditions at the critical point (coordinate $r_{c}$ and velocity $u_{c}$ ) are derived from the relations below, which are similar to those obtained in [14]:

$$
\begin{aligned}
& 4 u_{c}^{2}=\frac{r_{g}}{r_{c}}-\frac{2 Q^{2}}{r_{c}^{2}}, \\
& u_{c}^{2}=V_{c}^{2} F\left(r_{c}, u_{c}\right) .
\end{aligned}
$$

From these two equations, one obtains for the critical radius:

$$
r_{c}=\frac{r_{g}\left(1+3 V_{c}^{2}\right)}{8 V_{c}^{2}}\left\{1 \pm\left[1-\frac{8 V_{c}^{2} \beta^{2}\left(1+V_{c}^{2}\right)}{\left(1+3 V_{c}^{2}\right)^{2}}\right]^{1 / 2}\right\} .
$$

As pointed out by [13], two solutions for the critical radius are possible. The first corresponds to the sign “+”, which locates the critical radius outside the event horizon and represents a true physical solution. The other possibility corresponds to the sign "-" locates the critical radius between the inner and the outer horizon. This last solution will be discarded in the present analysis.

\section{Accretion of a Nonrelativistic Fluid}

For a baryonic nonrelativistic fluid with an equation of state $P=K n^{\gamma}$, the energy density is given by

$$
\varepsilon=m c^{2} n+\frac{P}{(\gamma-1)} .
$$

Notice that the first term on the right side of (16) corresponds to the rest energy (usually neglected) while the second represents the interaction energy among the fluid particles. Using these relations and (12), one obtains after some algebra:

$$
V^{2}=\frac{a^{2}}{\left[1+a^{2} /(\gamma-1)\right]},
$$

where $a^{2}=\gamma P / m n c^{2}$ is the square of the adiabatic sound velocity measured in units of the velocity of light. Notice that solutions with $\gamma=1$ are excluded since in this case we have a null velocity for the flow. The constant $\Delta$ in (9) can be calculated by imposing a zero flow velocity $(u=0)$ when $r \rightarrow \infty$ or, in other words, at distances far away from the influence sphere of the black hole. Under these conditions, one obtains trivially

$$
\Delta=m c^{2}\left[1+\frac{a_{\infty}^{2}}{(\gamma-1)}\right],
$$

where $a_{\infty}$ is the adiabatic sound velocity well beyond the influence radius of the black hole. Using this result and evaluating now (9) at the critical point with the help of (13), one obtains a relation between the adiabatic sound velocity at the critical point and that at "infinity," that is,

$$
\frac{a_{c}}{a_{\infty}} \approx \sqrt{\frac{2}{(5-3 \gamma)}} .
$$

The flow velocity at the critical point can be evaluated from (11), (14), and (17) under the approximation $a<<1$ (the sound velocity is much less than the velocity of light). Performing a series expansion up to third order, one obtains

$$
u_{c} \approx a_{c}-\frac{3}{4} \frac{(5-3 \gamma)}{(\gamma-1)} a_{c}^{3} .
$$

Inspection of this equation reveals two distinct and important aspects: firstly, as we have mentioned before, the flow velocity at the critical point is not exactly coincident with the sound velocity, since they differ at least by a term of third order in the sound velocity. Secondly, at the considered order, the flow velocity at the critical point is not affected by the black hole charge. However, this is not the case for the critical radius. Substituting the relations above into (15), one obtains for the critical radius up to second-order terms in $\beta$

$$
\frac{r_{c}}{r_{g}} \approx \frac{(5-3 \gamma)}{8 a_{\infty}^{2}}\left[1+\frac{\left(6-4 \beta^{2}\right) a_{\infty}^{2}}{(5-3 \gamma)}\right] .
$$

Notice that, as in the Schwarzschild case, the existence of a physical solution requires $\gamma<5 / 3$ and that the electrical charge reduces slightly the distance of the critical point with respect to the black hole horizon.

The accretion rate can now be computed from the conditions at the critical radius, that is,

$$
\frac{d M_{\mathrm{bh}}}{d t}=4 \pi r_{c}^{2} T_{t}^{r}=\pi \Gamma(\gamma)\left(G M_{\mathrm{bh}}\right)^{2} f(\beta) \rho_{\infty} a_{\infty}^{-3} .
$$

In the equation above, $\rho_{\infty}=m n_{\infty}$ is the mass density of the baryonic fluid at "infinity" (beyond the influence radius of the black hole), $M_{\mathrm{bh}}$ is the black hole mass, and the functions $\Gamma(\gamma)$ and $f(\beta)$ are defined, respectively, as

$$
\begin{aligned}
& \Gamma(\gamma)=\left[\frac{2}{(5-3 \gamma)}\right]^{(5-3 \gamma) / 2(\gamma-1)}, \\
& f(\beta)=\left[1+\frac{\left(12-8 \beta^{2}\right) a_{\infty}^{2}}{(5-3 \gamma)}\right] .
\end{aligned}
$$




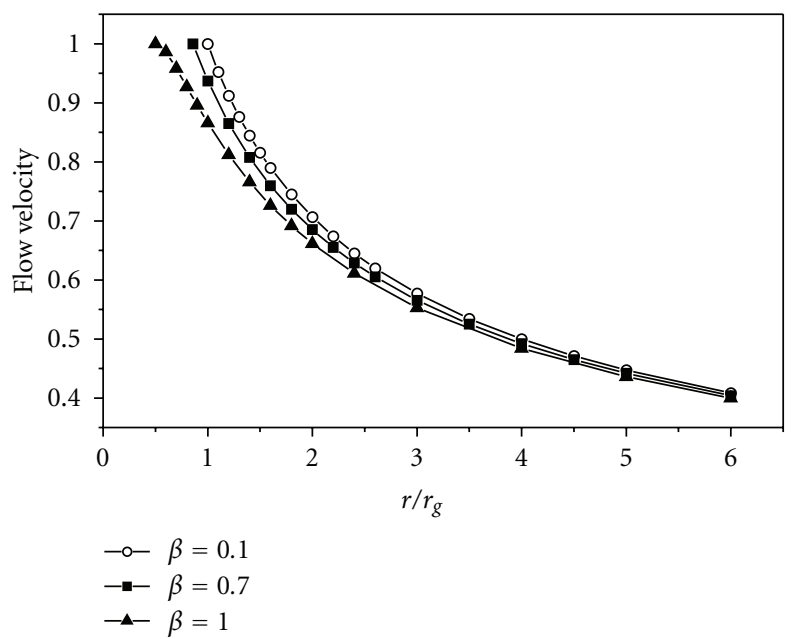

FIGURE 1: Radial velocity profiles for a nonrelativistic fluid being accreted by a Reissner-Nordström black hole for different chargeto-mass ratios. The radial coordinate is given in terms of the gravitational radius.

3.1. Some Numerical Results. In order to derive the radial velocity and the density profiles of the flow, let us define the dimensionless variables $x=r / r_{g}$ and $y=n / n_{\infty}$, which measure, respectively, the radial distance in terms of the gravitational radius $r_{g}$ and the particle number density in terms of its value at "infinity." Recall that the flow velocity $u$ is given in units of the velocity of light. Under these conditions, (9) can be written as

$$
\left(1+\lambda_{\infty}^{2} y^{\gamma-1}\right)\left(1-\frac{1}{x}+\frac{1}{4} \frac{\beta}{x^{2}}+u\right)^{1 / 2}=\left(1+\lambda_{\infty}^{2}\right),
$$

where $\lambda_{\infty}^{2}=a_{\infty}^{2} /(\gamma-1)$ and, as mentioned above, the adiabatic sound velocity is also given in terms of the velocity of light. Using the same notation, the particle density conservation (8) can be written as

$$
y=(\gamma-1)^{1 / 2} \lambda_{\infty}\left(\frac{x_{c}}{x}\right)^{2}\left(\frac{a_{c}}{a_{\infty}}\right)^{(\gamma+1) /(\gamma-1)} .
$$

The constant in (8) was calculated by applying the particle conservation equation at the critical point and using the previous result on the ratio between the adiabatic sound velocity at "infinity" and at the critical point. Equations (25) and (26) constitute an algebraic system of nonlinear equations, which can be solved numerically for the fluid velocity $u$ and the ratio $n / n_{\infty}$ for a given value of $x=$ $r / r_{g}$. The parameters characterizing the flow are the fluid temperature (or the sound velocity) at "infinity" and the adiabatic coefficient, while the parameter $\beta=Q / M$ affects the position of the critical point. The radial velocity profile of the flow (in units of the velocity of light) derived numerically from the equations above is shown in Figure 1. The velocity profile was computed by assuming a fluid temperature of $10^{4} \mathrm{~K}$ at "infinity" and an adiabatic coefficient $\gamma=1.2$, adequate for an ionized gas. Solutions were obtained for

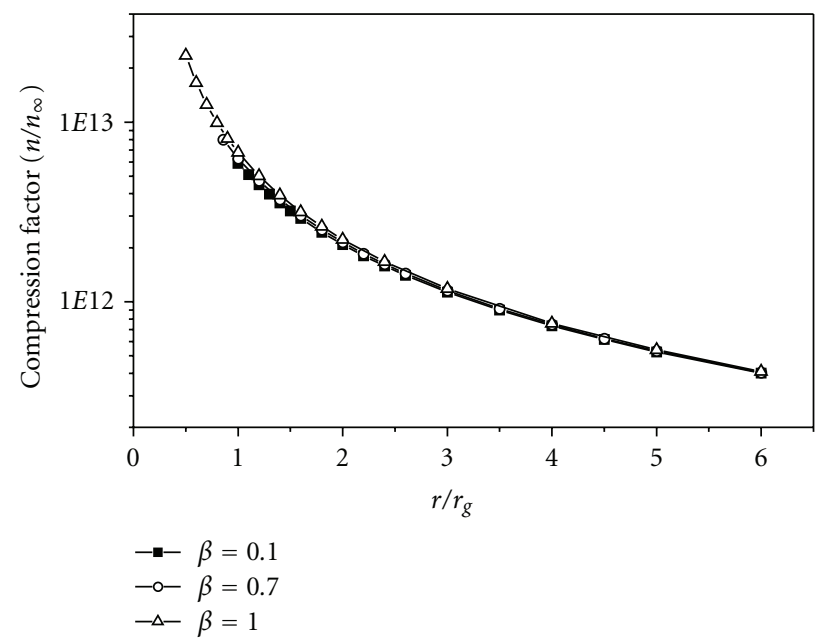

Figure 2: Compression factor profiles for an inflow of a nonrelativistic gas as a function of the radial coordinate in units of the gravitational radius for different charge-to-mass ratios.

different values of the charge, that is, $\beta=0.1,0.7$, and 1.0 (extreme Reissner-Nordström case). For these models, the event horizon is located, respectively, at $0.9975,0.8571$, and 0.5000 times the gravitational radius of a Schwarzschild black hole. Notice that for all these cases, when the charge-to-mass ratio is in the range $0<\beta \leq 1$, the flow crosses the event horizon always at the velocity of light. In these examples, the critical radius is very far from the event horizon $\left(r_{c} \approx\right.$ $\left.1.575 \times 10^{8} r_{g}\right)$ and the flow velocity at the critical point is about $12 \mathrm{kms}^{-1}$.

Figure 2 shows the particle density profile in terms of the density at "infinity" (compression ratio) as a function of the radial coordinate, measured in units of the gravitational radius and for different values of the charge-to-mass ratio. The compression ratio at the event horizon increases as $\beta$ increases, reaching a factor of about $2.352 \times 10^{13}$ for an extreme RN black hole. Since the temperature varies as $T \propto n^{\gamma-1}$, it may attain values of the order of $10^{6}-10^{7} \mathrm{~K}$ near the horizon. It should be emphasized that these values correspond to an adiabatic flow and that radiative transfer effects may change appreciably these results.

When $V_{c}<<1$ (nonrelativistic flow), (21) admits real solutions only if the charge-to-mass ratio satisfies the condition:

$$
\beta^{2} \leq \frac{\left(1+3 V_{c}^{2}\right)^{2}}{8 V_{c}^{2}\left(1+V_{c}^{2}\right)} \approx \frac{1}{8 V_{c}^{2}} .
$$

The extreme case $\beta^{2} \approx 1 /\left(8 V_{c}^{2}\right)>1$ corresponds to a situation of a naked singularity. The critical radius is located at $r_{c} \approx r_{g} /\left(8 V_{c}^{2}\right)$, close to the sonic point within the considered approximation. However, no solution for the flow beyond the critical point was found under these circumstances. In [13], the authors suggested that when $\beta>$ 1 , an ideal fluid does not accrete at all onto a naked singularity, instead a static "atmosphere" will be formed. In 
this case, the fluid should obey the hydrostatic equilibrium equation:

$$
\frac{\partial P}{\partial r}+(P+\varepsilon) \frac{\partial \lg \sqrt{B(r)}}{\partial r}=0 .
$$

The solution of this equation indicates that the matter density increases as the singularity is approached, attains a maximum, and then decreases toward the limit $\rho \rightarrow 0$ as $r \rightarrow 0$. This configuration, including a density inversion, is clearly unstable (Rayleigh-Taylor instability). The observed behaviour can be explained by the fact that the effective acceleration $g_{e f}=\partial \lg \sqrt{B(r)} / \partial r$ is always negative outside the horizon but becomes positive for $r<0.5 \beta^{2} r_{g}$. The repulsive force due to the modification of the space curvature near the singularity is a consequence of the presence of the electric charge. Past investigations [18] on the gravitational collapse of a uniform sphere constituted by charged dust led to a similar conclusion. Once the horizon is crossed by the surface of the sphere, the gravitational attraction is replaced by a repulsive force and, consequently, the surface of the sphere never reaches the singularity. Therefore, in a certain sense, the singularity is "protected" by the repulsive force induced by the electric charge.

\section{Accretion of a Relativistic Fluid}

In the case of a relativistic fluid, the equation of state is simply given by $P=\varepsilon / 3$ and the pressure is related to the particle number density by $P \propto n^{4 / 3}$. Under these conditions, from (12), one obtains trivially that $V^{2}=1 / 3$. Replacing this result into (15), one obtains for the critical radius:

$$
r_{c}=\frac{3}{4} r_{g}\left[1 \pm\left(1-\frac{8}{9} \beta^{2}\right)^{1 / 2}\right] .
$$

This relation indicates that a critical point exists only if $\beta \leq 3 / \sqrt{8}$. This leaves open the possibility for the existence of relativistic flows in the presence of a naked singularity. However, even in this case, no solutions were found when $\beta>1$ for the same reasons already mentioned.

When $\beta=0$ (the case of a Schwarzschild black hole), contrary to what happens for a nonrelativistic fluid, the critical radius is located near the horizon, that is, $r_{c} / r_{g}=3 / 2$ and the radial velocity of the flow at this point is $u_{c}=1 / \sqrt{6}$, implying that the crossing occurs subsonically. The flow becomes supersonic only at $r_{s} \approx 1.07754 r_{g}$, quite close to the horizon, which is crossed with a velocity of $\sim 0.62 \mathrm{c}$. Using (9) and the conditions at the critical point, the ratio between the density or the temperature $\left(T \propto n^{1 / 3}\right)$ at this point and the value at "infinity" can be easily computed, for example,

$$
\left(\frac{n_{c}}{n_{\infty}}\right)^{1 / 3}=\left(\frac{T_{c}}{T_{\infty}}\right)=\sqrt{2}
$$

When $\beta<<1$, the critical radius is given approximately by

$$
r_{c} \approx \frac{3}{2} r_{g}\left(1-\frac{2}{9} \beta^{2}\right)
$$

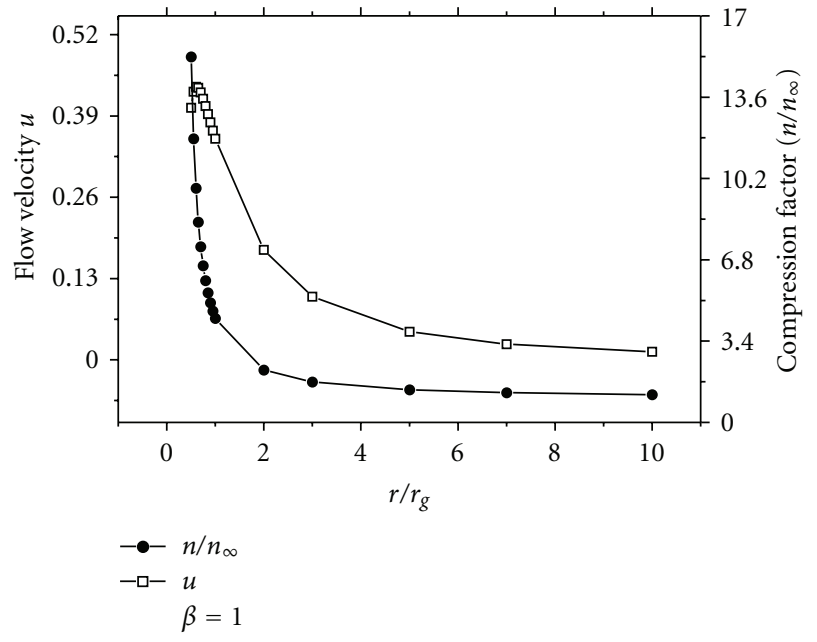

FIGURE 3: The radial velocity profile (left ordinate) for an accreting relativistic fluid as a function of the radial coordinate, in units of the gravitational radius, for an extreme RN black hole. The profile of the compression ratio (right ordinate) is also shown.

Using this result and (13), the flow velocity at the critical point is

$$
u_{c}^{2} \approx \frac{1}{6}\left(1-\frac{1}{9} \beta^{2}\right)
$$

Notice that the flow velocity at the critical point is reduced by the effect of the charge and the critical radius approaches the horizon.

For the extreme case $(\beta=1)$, two solutions for the critical radius exist. The first locates the critical radius at a distance twice the horizon and the flow velocity at this point is $u_{c}=1 / \sqrt{8}$. The other solution is not physically acceptable, since the critical radius coincides with the horizon, resulting in a null flow velocity at this position. The velocity and the density profiles of the flow can be derived as explained in Section 3.1, taking into account that for a relativistic fluid $(P+\epsilon) / n \propto n^{1 / 3}$. In Figure 3, the radial velocity profile is shown as a function of the radial coordinate given in units of the gravitational radius for an extreme RN black hole. After crossing the critical point, the flow reaches a maximum velocity of about $u_{\max } \approx 0.4367$ at $r_{\max } \approx 0.60 r_{g}$. Then the velocity decreases and the flow crosses the horizon with a subsonic velocity of about 0.4029 c. Compression ratios attained by a relativistic fluid are always considerably smaller than those derived for the nonrelativistic case. For an extreme $\mathrm{RN}$ black hole, the compression factor near the horizon is about 15.3, about twelve orders of magnitude less than that obtained for a nonrelativistic fluid. Since in the relativistic case, the temperature varies as $T \propto n^{1 / 3}$, near the horizon, due to the adiabatic compression, the temperature is only about 2.48 times the value at "infinity."

The accretion rate can be computed from the flow conditions at the critical point by following the same steps as before. In this case, one obtains

$$
\frac{d M_{\mathrm{bh}}}{d t}=32 \sqrt{3} \pi \frac{\left(G M_{\mathrm{bh}}\right)^{2}}{c^{5}} \varepsilon_{\infty}\left(1+O\left(\beta^{4}\right)\right),
$$


where $\varepsilon_{\infty}$ is the energy density of the fluid at "infinity." This relation differs from the Schwarzschild case only in terms of the order of $\beta^{4}$. It is worth mentioning that the accretion rate of relativistic particles by a black hole is usually computed by using the capture cross-section $\sigma_{\text {cap }}=27 \pi r_{g}^{2} / 4$, leading to a numerical factor in (33) equal to 27 instead of $32 \sqrt{3}$ here obtained. The simple use of the capture cross-section neglects hydrodynamical and relativistic effects that increase the accretion efficiency by about a factor of two. In the case of an extreme RN black hole, the accretion rate is given by

$$
\frac{d M_{\mathrm{bh}}}{d t}=\frac{512 \sqrt{3} \pi}{27} \frac{\left(G M_{\mathrm{bh}}\right)^{2}}{c^{5}} \varepsilon_{\infty} .
$$

It can be easily verified that in this case the accretion efficiency decreases by almost $60 \%$ with respect to an uncharged black hole.

\section{Conclusions}

The steady and spherically symmetric accretion of nonrelativistic and relativistic fluids into a Reissner-Nordström black hole was revisited. A charged black hole modifies slightly the properties of the inflow of a nonrelativistic fluid since the critical radius, the radial velocity at the critical point, and the accretion rate are affected only by second-order terms in the charge-to-mass ratio.

The situation is rather different for the inflow of relativistic fluids. Firstly, the critical point occurs closer to the horizon, what is not the case for the inflow of nonrelativistic fluids. Secondly, the crossing of the critical point occurs always in a subsonic regime, even if the black hole is uncharged, contrary to what is usually stated in the literature. The sonic point is reached only very near the horizon and the fluid crosses the horizon with a velocity less than the light speed. In nonrelativistic flows, the ratio between the particle density near the horizon and at "infinity" may attain values of the order of $10^{13}$, if the flow is adiabatic. This is not the case when relativistic flows are considered, since in this situation, the compression ratio varies from about 2.83 for a weakly charged black hole up to 4.35 for the extreme case $(\beta=1)$. Finally, the accretion rate of a relativistic fluid by an extreme RN black hole is about $60 \%$ smaller than that expected for a Schwarzschild black hole.

When $\beta>1$, the singularity is "naked" and, depending on the value of the charge-to-mass ratio, mathematical solutions for the critical point exist either for nonrelativistic or for relativistic fluids. However, no solutions for the flow beyond the critical point were found. Some authors [13] suggested that beyond that point, a "static" solution is possible. These solutions present an inversion in the mass density profile, consequence of a repulsive force due to the black hole charge, which is manifested for distances less than $\beta^{2} G M_{\mathrm{bh}} / c^{2}$. Such an inversion observed in the mass density profile is probably unstable against the Rayleigh-Taylor instability, suggesting that such static solutions cannot exist.

\section{References}

[1] S. K. Chakrabarti, "Accretion processes on a black hole," Physics Report, vol. 266, no. 5-6, pp. 229-390, 1996.

[2] F. C. Michel, "Accretion of matter by condensed objects," Astrophysics and Space Science, vol. 15, no. 1, pp. 153-160, 1972.

[3] S. Peirani and J. A. de Freitas Pacheco, "Dark matter accretion into supermassive black holes," Physical Review D, vol. 77, no. 6, Article ID 064023, 2008.

[4] J. P. Ostriker, R. McCray, R. Weaver, and A. Yahil, "A new luminosity limit for spherical accretion onto compact X-ray sources," The Astrophysical Journal, vol. 208, pp. L61-L65, 1976.

[5] P. Meszaros, "Radiation from spherical accretion onto black holes," Astronomy \& Astrophysics, vol. 44, pp. 59-68, 1975.

[6] K. S. Thorne, R. A. Flammang, and A. N. Zytkow, "Stationary spherical accretion into black holes-I: equations of structure," Monthly Notices of the Royal Astronomical Society, vol. 194, pp. 475-484, 1981.

[7] R. A. Flammang, "Stationary spherical accretion into black holes-II: theory of optically thick accretion," Monthly Notices of the Royal Astronomical Society, vol. 199, pp. 833-867, 1982.

[8] M. G. Park, "Self-consistent models of spherical accretion onto black holes. II. Two-temperature solutions with pairs," The Astrophysical Journal, vol. 354, no. 1, pp. 83-97, 1990.

[9] L. Nobili, R. Turolla, and L. Zampieri, "Spherical accretion onto black holes: a complete analysis of stationary solutions," The Astrophysical Journal, vol. 383, no. 1, pp. 250-262, 1991.

[10] T. Di Matteo, N. Khandai, C. DeGraf et al., "Cold gas flows and the first quasars in cosmological simulations," http://arxiv .org/abs/1107.1253v1.

[11] C. Cherubini, A. Geralico, J. A. Rueda, and R. Ruffini, " $e^{-} e^{+}$ pair creation by vacuum polarization around electromagnetic black holes," Physical Review D, vol. 79, no. 12, Article ID 124002, 15 pages, 2009.

[12] J. A. de Freitas Pacheco and J. E. Horvath, "Generalized second law and phantom cosmology," Classical and Quantum Gravity, vol. 24, no. 22, pp. 5427-5433, 2007.

[13] E. Babichev, V. I. Dokuchaev, and Yu. N. Eroshenko, "Perfect fluid and scalar field in the Reissner-Nordstrom metric," Journal of Experimental and Theoretical Physics, vol. 112, pp. 784-793, 2011.

[14] M. Jamil, A. Qadir, and M. A. Rashid, "Charged black holes in phantom cosmology," European Physical Journal C, vol. 58, no. 2, pp. 325-329, 2008.

[15] D. N. Page, "Evidence against macroscopic astrophysical dyadospheres," The Astrophysical Journal, vol. 653, no. 2, pp. 1400-1409, 2006.

[16] J. I. Castor, D. C. Abbott, and R. I. Klein, "Radiation-driven winds in of stars," The Astrophysical Journal, vol. 195, pp. 157$174,1975$.

[17] F. X. Araujo and J. A. de Freitas Pacheco, "Radiatively driven winds with azimuthal symmetry-application to Be stars," Monthly Notices of the Royal Astronomical Society, vol. 241, pp. 543-557, 1989.

[18] I. Novikov, "The replacement of relativistic gravitational contraction by expansion, and the physical singularities during contraction," Soviet Astronomy. AJ, vol. 10, no. 5, p. 731, 1967. 

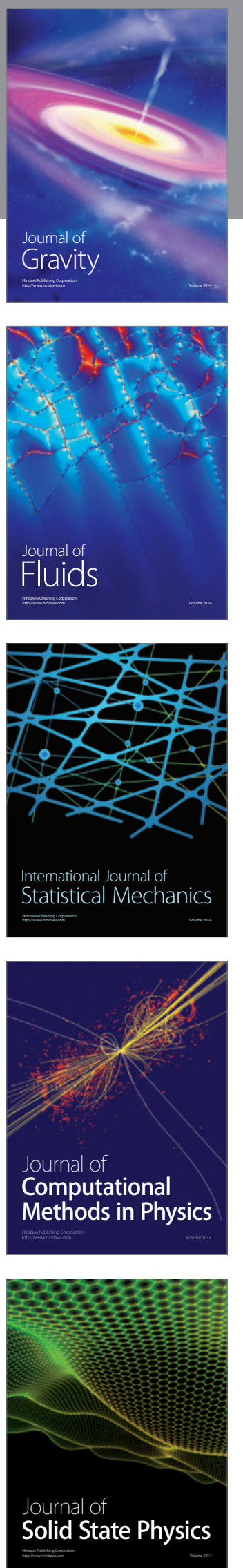

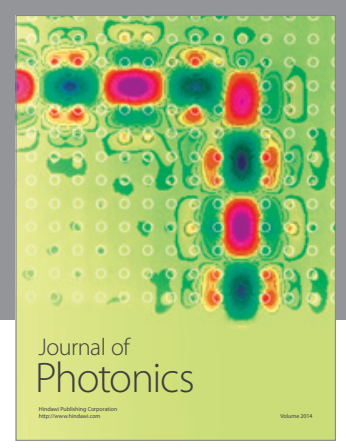

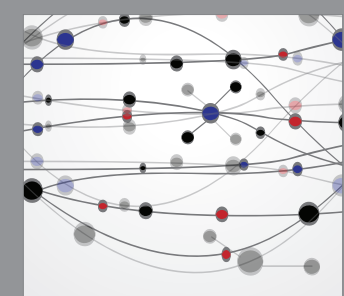

The Scientific World Journal
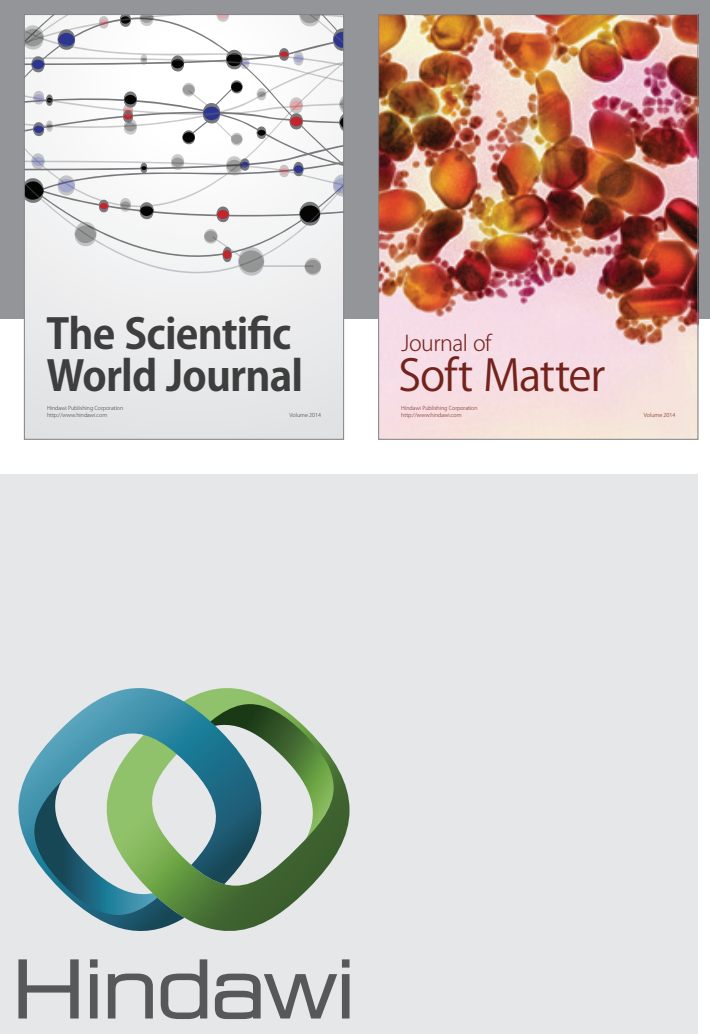

Submit your manuscripts at

http://www.hindawi.com
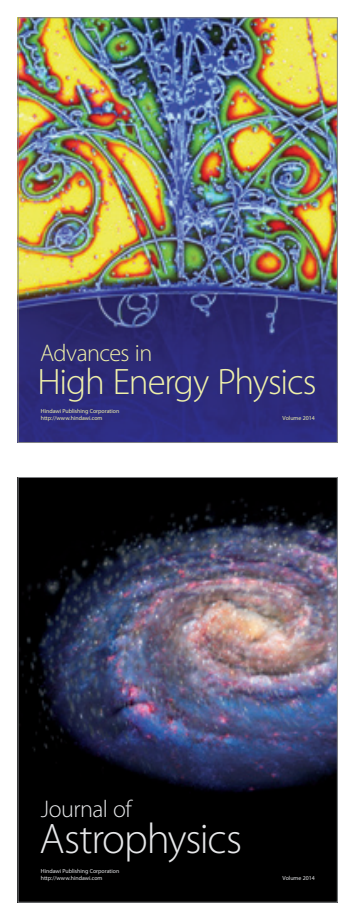
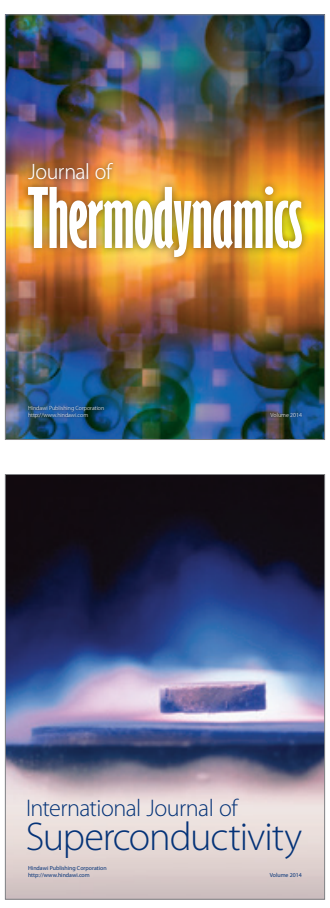
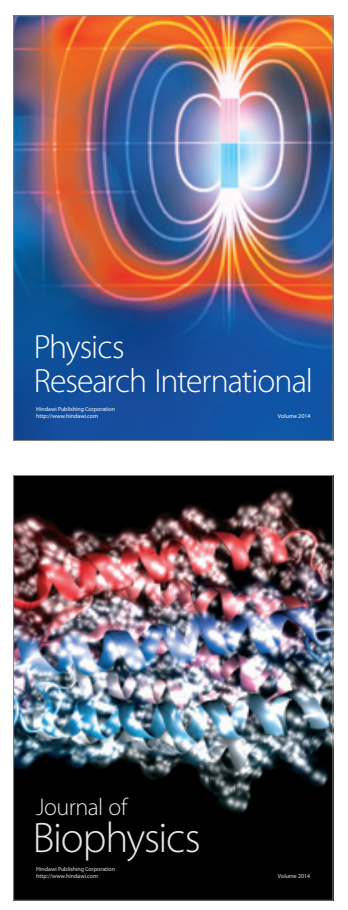
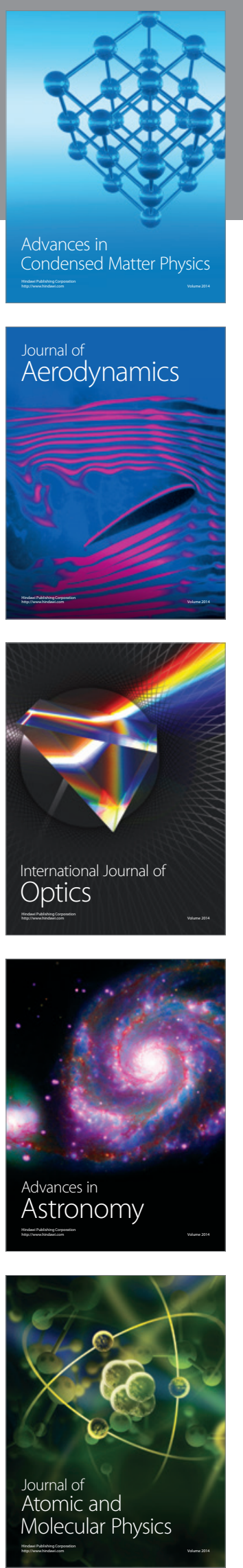